Acknowledgements: This work has been supported by a research grant from FOREUM Foundation for Research in Rheumatology.

Disclosure of Interests: Anne Kerola Speakers bureau: Boehringer-Ingelheim, Consultant of: Pfizer, Gilead, Boehringer-Ingelheim, Joseph Sexton: None declared, Grunde Wibetoe: None declared, Silvia Rollefstad: None declared, Cynthia S. Crowson: None declared, Espen Haavardsholm: None declared, Tore K. Kvien Speakers bureau: Amgen, Celltrion, Egis, Evapharma, Ewopharma, Hikma, Oktal, Sandoz, Sanofimgen, Celltrion, Egis, Evapharma, Ewopharma, Hikma, Oktal, Sandoz, Sanofi, Consultant of: AbbVie, Amgen, Biogen, Celltrion, Eli Lilly, Gilead, Mylan, Novartis, Pfizer, Sandoz, Sanofi, Grant/research support from: research funding to Diakonhjemmet Hospital from AbbVie, Amgen, BMS, MSD, Pfizer and UCB, Anne Grete Semb Speakers bureau: AbbVie, Bayer, Lilly, Novartis, and Sanofi, Consultant of: Sanofi, Grant/research support from: collaborative research support from Lilly, outside the submitted work.

DOI: 10.1136/annrheumdis-2021-eular.975

\section{POS0030 SARCOIDOSIS INCIDENCE IN A NORTHERN SPANISH HEALTH REGION.}

C. Álvarez-Reguera ${ }^{1}$, R. Fernández-Ramón², J. J. Gaitán-Valdizán², J. L. Martín-Varillas ${ }^{3}$, L. Sanchez-Bilbao ${ }^{1}$, D. Martínez-López ${ }^{3}$, I. GonzálezMazón ${ }^{1}$, R. Demetrio-Pablo², M. Á. González-Gay ${ }^{1}$, R. Blanco ${ }^{1} .{ }^{1}$ Hospital Universitario Marqués de Valdecilla, Rheumatology, Santander, Spain; ${ }^{2}$ Hospital Universitario Marqués de Valdecilla, Ophthalmology, Santander, Spain;

${ }^{3}$ Hospital de Sierrallana, Rheumatology, Torrelavega, Spain

Background: Sarcoidosis is a systemic and potentially severe disease (1). Its incidence varies widely worldwide.

Objectives: The aim of this study was to estimate the sarcoidosis incidence in a Northern Spanish population-based cohort.

Methods: All incident cases of sarcoidosis between January 1999 and December 2019 in a tertiary hospital were reviewed. Inclusion criteria were a) diagnosis of sarcoidosis according to ATS/ERS/WASOG (Eur Respir J. 1999; 14:735-7) and b) patients residing in our health region. Incidence between 1999 and 2019 was estimated by sex, age, and year of diagnosis.

Results: From a total of 384 patients diagnosed with sarcoidosis, 234 (129 women/ 105 men) met the inclusion criteria and were finally included in the study. Mean age of the cohort at diagnosis was $48.43 \pm 14.83$ years $(46.95 \pm 14.50$ in males; $49.63 \pm 15.04$ in females, $p=0.12$ ). Annual incidence during 1999-2019 period was 3.56 per 100,000 population (3.32 per 100,000 in males and 3.72 per 100,000 in females). An upward trend in annual incidence over time was observed with rates ranging from 1.73 per 100,000 inhabitants in 1999 to 6.91 per 100,000 inhabitants in 2016 (Figure 1). Overall, sarcoidosis was predominantly diagnosed during middle adulthood. A bimodal distribution of age-specific incidence rates was observed in both sexes with two peaks in the age groups of 30-39 $(4.98$ per 100,000) and $60-69$ years $(5.12$ per 100,000$)$ in men, and in $40-49(6.45$ per 100,000$)$ and 60-69 years $(5.94$ per 100,000$)$ in women.

Comparative studies with other regions are summarize in Table 1.

Conclusion: A progressive increase in incident sarcoidosis is observed. The estimated incidence of sarcoidosis in this study $(3.56$ per 100,000) was like that of other Mediterranean countries (2). No gender predominance was observed. Demographics variations, changes in practice patters or diagnostic test improvements could explain the upward trend in sarcoidosis incidence detected in our study. Consistent with previous studies, male presented an incidence peak 10 years earlier than female (3-5).

\section{REFERENCES:}

[1] Riancho-Zarrabeitia L, et al. Clin Exp Rheumatol 2014; 32(2): 275-84. PMID: 24321604

[2] Brito-Zerón P, et al. Clin Exp Rheumatol. 2019;37(6):1052-64.

[3] Arkema E V., et al. Eur Respir J. 2016;48(6):1690-9.

[4] Yoon H, et al. Am J Respir Crit Care Med. 2018;197(MeetingAbstracts):1-8.

[5] Ungprasert P, et. Mayo Clin Proc. 2016 Feb;91(2):183-8.
A

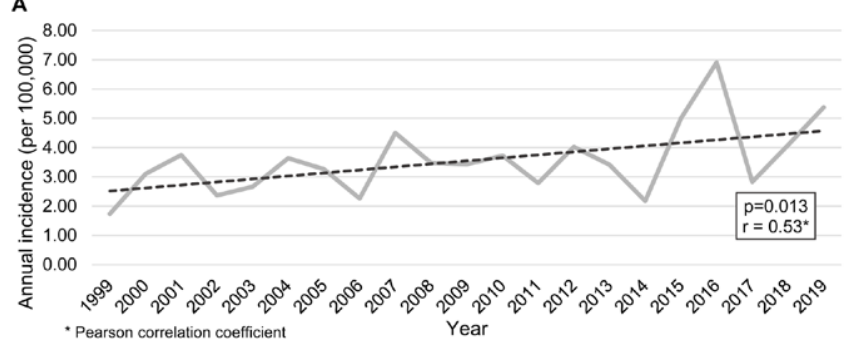

Figure 1. Annual incidence of sarcoidosis in 1999-2019 (A)

Table 1. Sarcoidosis incidence rates reported in the literature.

\begin{tabular}{|c|c|c|c|c|}
\hline Study, year & Country, data source & Time period & Incident cases & $\begin{array}{c}\text { Incidence } \\
\text { per 100,000 } \\
\text { persons-year }\end{array}$ \\
\hline Pietinalho, 1995 & $\begin{array}{c}\text { Finland, hospital } \\
\text { database }\end{array}$ & 1984 & 1,378 & 11.4 \\
\hline Pietinalho, 1995 & $\begin{array}{l}\text { Japan, Hokkaido, hospital } \\
\text { database }\end{array}$ & 1984 & 288 & 1.0 \\
\hline Yigla, 2002 & Israel, hospital database & 1980-1996 & 120 & 0.8 \\
\hline Byg, 2003 & $\begin{array}{l}\text { Denmark, Danish } \\
\text { National Patient } \\
\text { Registry }\end{array}$ & 1980-1994 & 5,536 & 7.2 \\
\hline Gribbin, 2006 & $\begin{array}{l}\text { UK, Health Improvement } \\
\text { Network }\end{array}$ & $1990-2003$ & 1,019 & 5.0 \\
\hline Gillman, 2007 & $\begin{array}{c}\text { Australia, Victoria, hospi- } \\
\text { tal database }\end{array}$ & $1995-2005$ & 122 & $4.4-6.3$ \\
\hline $\begin{array}{l}\text { Haraldsdottir, } \\
\quad 2007\end{array}$ & $\begin{array}{c}\text { Iceland, hospital } \\
\text { database }\end{array}$ & $1981-2003$ & 235 & 3.8 \\
\hline Musellin, 2009 & $\begin{array}{c}\text { Turkey, healthcare } \\
\text { providers }\end{array}$ & $2004-2006$ & 293 & 4.0 \\
\hline $\begin{array}{l}\text { Deubelbeiss, } \\
2010\end{array}$ & $\begin{array}{c}\text { Switzerland, Swiss } \\
\text { Federal Office for } \\
\text { Statistics }\end{array}$ & $2002-2005$ & 2,925 & 7.0 \\
\hline Kowalska, 2014 & $\begin{array}{l}\text { Poland, Katowice, } \\
\text { National Health Fund }\end{array}$ & 2006-2010 & 1,217 & $3.8-4.5$ \\
\hline Arkema, 2016 & $\begin{array}{c}\text { Sweden, National Patient } \\
\text { Register }\end{array}$ & 2003-2013 & 10,787 & $10.4-14.8$ \\
\hline $\begin{array}{l}\text { Baughman, } \\
2016\end{array}$ & $\begin{array}{c}\text { USA, Optum Health Care } \\
\text { Database }\end{array}$ & $2010-2013$ & 29,372 & $\begin{array}{c}\text { African American: } \\
\text { 17.8; Caucasian: } \\
\text { 8.1; Hispanic: } \\
\text { 4.3; Asian: } 3.2\end{array}$ \\
\hline $\begin{array}{l}\text { Duchemann, } \\
2017\end{array}$ & $\begin{array}{c}\text { France, Seine-Saint- } \\
\text { Denise County }\end{array}$ & 2012 & 361 & 4.9 \\
\hline $\begin{array}{l}\text { Škopljanac, } \\
2017\end{array}$ & $\begin{array}{c}\text { Croatia, Split- Dalmatia } \\
\text { County, hospital } \\
\text { database }\end{array}$ & 1986-2015 & 318 & $3.1-3.4$ \\
\hline Yoon, 2018 & $\begin{array}{c}\text { Korea, National Health } \\
\text { Insurance }\end{array}$ & $2007-2016$ & 4,791 & 0.85 \\
\hline Fidler, 2019 & $\begin{array}{l}\text { Canada, Ontario, health } \\
\text { administrative data }\end{array}$ & 1996-2015 & 18,550 & $6.8-7.9$ \\
\hline $\begin{array}{l}\text { Fernandez, } \\
\quad 2011\end{array}$ & $\begin{array}{c}\text { Spain, Leon, hospital } \\
\text { database }\end{array}$ & $2001-2008$ & 118 & 4.51 \\
\hline $\begin{array}{l}\text { Present study, } \\
2020\end{array}$ & $\begin{array}{c}\text { Spain, Santander, hospi- } \\
\text { tal database }\end{array}$ & 1999-2019 & 234 & 3.56 \\
\hline
\end{tabular}

Disclosure of Interests: Carmen Álvarez-Reguera: None declared, Raúl Fernández-Ramón: None declared, Jorge Javier Gaitán-Valdizán: None declared, José Luis Martín-Varillas: None declared, Lara Sanchez-Bilbao: None declared, David Martínez-López: None declared, Iñigo González-Mazón: None declared, Rosalía Demetrio-Pablo: None declared, Miguel Á. González-Gay Speakers bureau: Abbvie, Pfizer, Roche, Sanofi and MSD., Grant/research support from: Abbvie, MSD, Jansen and Roche, Ricardo Blanco Speakers bureau: Abbvie, Pfizer, Roche, Bristol-Myers, Janssen, Lilly and MSD., Grant/research support from: Abbvie, MSD and Roche. DOI: 10.1136/annrheumdis-2021-eular.1870 